\title{
Short hairpin RNA treatment improves gait in a mouse model of Charcot-Marie-Tooth disease type $1 \mathrm{~A}$
}

\author{
HYUN MYUNG DOO ${ }^{1,2^{*}}$, YOUNG BIN HONG ${ }^{3 *}$, JIYOU HAN $^{4}$, HYO WON MOON $^{5}$, \\ HYUN HWANG $^{5}$, GEON KWAK ${ }^{1}$, SOO HYUN NAM ${ }^{2}$, SANG BEOM KIM ${ }^{6}$, KI WHA CHUNG ${ }^{7}$, \\ JONG HYUN KIM ${ }^{4}$ and BYUNG-OK CHOI ${ }^{1,2,5}$
}

\author{
${ }^{1}$ Department of Health Sciences and Technology, Samsung Advanced Institute for Health Science and Technology (SAIHST), \\ Sungkyunkwan University; ${ }^{2}$ Stem Cell and Regenerative Medicine Institute, Samsung Medical Center, Seoul 06351; \\ ${ }^{3}$ Department of Biochemistry, College of Medicine, Dong-A University, Busan, South Gyeongsang 49201; \\ ${ }^{4}$ Department of Biological Sciences, Laboratory of Stem Cell Research and Biotechnology, \\ Hyupsung University, Hwasung-si, Gyeonggi 18330; ${ }^{5}$ Department of Neurology, \\ Samsung Medical Center, Sungkyunkwan University School of Medicine, Seoul 06351; ${ }^{6}$ Department of Neurology, \\ Kyung Hee University Hospital at Gangdong, Kyung Hee University School of Medicine, Seoul 05278; \\ ${ }^{7}$ Department of Biological Sciences, Kongju National University, Gongju, Chungcheong 32588, Republic of Korea
}

Received March 17, 2020; Accepted July 22, 2020

DOI: $10.3892 / \mathrm{mmr} .2020 .11579$

\begin{abstract}
Charcot-Marie-Tooth disease (CMT) is the most common inherited neurological disorder of the peripheral nervous system. The major subtype, CMT type 1A (CMT1A), accounts for $\sim 40 \%$ of CMT cases and is characterized by distal muscle atrophy and gait disturbances. Short hairpin (sh) RNA sequences are potentially advantageous therapeutic tools for distal muscle atrophy-induced gait disturbance. Therefore, the current study focused on the effects of an optimal shRNA injection using the myostatin (mstn) gene inhibition system. shLenti-Mstn A demonstrated significant suppression of endogenous mstn gene expression (>40\%) via RT-qPCR following direct injection into the gastrocnemius and rectus femoris of the hind limb in $\mathrm{C} 22$ mice. The results also reported that shLenti-Mstn A treatment increased muscle mass and size of the hind limbs compared with mock-treated mice via measurement of the mass of injected muscles and magnetic resonance imaging study. Furthermore, electrophysiological
\end{abstract}

Correspondence to: Dr Byung-Ok Choi, Department of Neurology, Samsung Medical Center, Sungkyunkwan University School of Medicine, 81 Irwon Road, Seoul 06351, Republic of Korea E-mail: bochoi@skku.edu

Dr Jong Hyun Kim, Department of Biological Sciences, Laboratory of Stem Cell Research and Biotechnology, Hyupsung University, 72 Choerubaek Road, Hwasung, Gyeonggi 18330, Republic of Korea E-mail:kimjh0446@naver.com

\section{*Contributed equally}

Key words: Charcot-Marie-Tooth disease, gait, muscle regeneration, short hairpin RNA, myostatin measurement using a Nicolet Viking Quest device revealed significantly improved compound muscle action potential (CMAP) in shLenti-Mstn A-treated mice compared with the mock group $(\mathrm{P}<0.05)$ whereas nerve conduction velocity $(\mathrm{NCV})$ showed no difference between groups. The shLenti-Mstn A treatment directly affected increased muscle regeneration, including mass and size, but not regeneration of peripheral nerve. Additionally, shLenti-Mstn A treatment significantly enhanced mobility, including locomotor coordination $(\mathrm{P}<0.01)$ and grip strength of the hindlimbs $(\mathrm{P}<0.01)$. Furthermore, MotoRater analysis using real-time recording with a high-speed camera revealed that shLenti-Mstn-treated mice exhibited an improved walking pattern in terms of step length, base support and duty factor compared with the mock group. It was hypothesized that treatment with shLenti-Mstn A may provide a novel therapeutic strategy for improving gait in patients with CMT1A.

\section{Introduction}

Gait disorders are commonly caused by peripheral nerve and muscle weakness due to the external environment or hereditary factors (1). Skeletal muscles are connected to the motor axons of peripheral nerves through neuromuscular junctions that control body movement (2). Peripheral neuropathy causes damage to nerves and muscles, leading to gait disturbances (3-5).

Previous studies have reported that hereditary peripheral neuropathy causes proximal and distal muscle atrophy due to axonal loss in motor nerves in murine models (6-8). However, the majority of current neuromuscular disease therapies are focused on regenerating axon nerves and myelination, not on muscle regeneration (3,9-11). For instance, a candidate drug for Schwann cell myelination improves the phenotype of peripheral neuropathy, motor nerve electrophysiology and 
locomotor coordination $(10,11)$. However, the drug does not directly improve muscle regeneration or gait pattern.

In vivo gene targeting therapies provide stronger therapeutic effects in the treatment of hereditary diseases compared with drug therapies $(12,13)$. A clustered regularly interspaced short palindromic repeats (CRISPR)/caspase 9-based therapy demonstrated a significant improvement in peripheral neuropathy following direct intraneural CRISPR-ribonucleoprotein injection into the sciatic nerve immediately distal to the sciatic notch in a C2C12 murine model with Charcot-Marie-Tooth disease type 1A (CMT1A) (13). The peripheral myelin protein 22 (PMP22)-overexpressing murine model C22 presents with severe demyelinating neuropathy, including muscle weakness and sensory depression, similar to symptoms exhibited by patients with CMT 1A. These mice usually develop CMT symptoms at 3 weeks of age (14). However, in the previous study, treatment for neuropathy did not improve gait regeneration (12).

Myostatin (Mstn) serves crucial roles in muscle regeneration and embryonic skeletal myogenesis $(15,16)$. Previous studies have demonstrated that Mstn inhibition is a strong potential candidate for the treatment of neuromuscular disease, including Duchenne muscular dystrophy (17-19). These results strongly indicated that adeno-associated virus-mediated Mstn inhibition improved muscle regeneration in terms of muscle mass and size. Another previous study reported that insulin-like growth factor-1 stimulates muscle hypertrophy by suppressing the Mstn signaling pathway (20). Therefore, it has been hypothesized that regulating Mstn expression may treat neuromuscular diseases, including amyotrophic lateral sclerosis (ALS), neuromuscular junction disorders and peripheral neuropathy.

In murine models of diseases, conventional behavioral tests, including the rotarod performance test, hind-limb grip strength test and footprint analysis, are useful tools for examining therapeutic effects on the central nervous system and peripheral nervous system (PNS) (7-11). Animal studies have attempted to analyze walking patterns using digital recording systems (21-23). For instance, in an ALS disease mouse model (23), the DigiGait ${ }^{\mathrm{TM}}$ Imaging System measures step length and $>30$ gait-related traits, including stance duration, swing duration and stance width $(21,22)$. Therefore, the DigiGait ${ }^{\mathrm{TM}}$ Imaging System may be a valuable tool for developing novel therapies to treat abnormal gait diseases.

The present study aimed to improve gait disorders using short hairpin (sh)RNA shLenti-Mstn therapy and evaluate effectiveness in the CMT1A mouse model using a digital gait system. RNA-based gene silencing, specifically using shRNAs, is a valuable tool and has clinical applications for target validation and therapeutics $(24,25)$.

\section{Materials and methods}

Cell culture. Mouse myoblast C2C12 (cat. no. CRL-1772) and 293T (cat. no. CRL-11268) cell lines were purchased from the American Type Culture Collection. C2C12 and 293T cells were cultured in DMEM with high glucose supplemented with $10 \% \mathrm{FBS}, 100 \mathrm{U} / \mathrm{ml}$ penicillin and $100 \mu \mathrm{g} / \mathrm{ml}$ streptomycin (all from Thermo Fisher Scientific, Inc.). Cultures were maintained at $37^{\circ} \mathrm{C}$ at $5 \% \mathrm{CO}_{2}$. $\mathrm{C} 2 \mathrm{C} 12$ cells were differentiated into myotubes by replacing the growth medium with a medium containing $2 \%$ horse serum (Thermo Fisher Scientific, Inc.) with $1 \%$ penicillin-streptomycin (Thermo Fisher Scientific, Inc.) and $1 \mathrm{X}$ insulin-transferrin-selenium supplement (Sigma-Aldrich; Merck KGaA).

In vitro transfection and selection of allele-specific shRNAs. Allele-specific shRNAs and control shRNAs were purchased from OriGene Technologies, Inc. A total of five vectors (one p-green fluorescence protein (GFP)-V-mock and four different shMstn sequences; $4 \mu \mathrm{g} /$ well) were transfected into $\mathrm{C} 2 \mathrm{C} 12$ myoblasts using Lipofectamine ${ }^{\circledR} 3000$ (Invitrogen; Thermo Fisher Scientific, Inc.), according to the manufacturer's protocol. The four different shRNAs contained the following sequences: shRNA-Mstn A, 5'-TGGCTCTTGAAGATGACG ATTATCACG-3'; B, 5'-ACAATCATTACCATGCCTACA GAGTCTGA-3'; C, 5'-CAACAGTGTTTGTGCAAATCC TGAGACTC-3'; and D, 5'-AATTCCAGCCATGGTAGTAGA CCGCTGTG-3'. To differentiate from myoblast cell to nascent myotubes, growth medium was replaced the following day and for five additional days with fresh medium containing $2 \%$ horse serum and $1 \%$ penicillin-streptomycin in DMEM with high glucose. At 7 days post-transfection, allelic specificity of the shRNAs was determined by comparing the expression of mstn mRNA in each transfected group using reverse transcription-quantitative PCR (RT-qPCR).

RNA isolation and cDNA synthesis. To quantify mRNA levels in the cell lines and lentivirus injected tissues, total mRNA was prepared for RT using an RNeasy Mini kit (Qiagen $\mathrm{GmbH}$ ), according to the manufacturer's protocol. mRNA concentrations were determined by optical density as measured by a spectrophotometer (NanoDrop 2000; Thermo Fisher Scientific, Inc.). Total mRNA $(2 \mu \mathrm{g})$ was used for cDNA synthesis using SuperScript ${ }^{\mathrm{TM}}$ II reverse transcriptase (Invitrogen; Thermo Fisher Scientific, Inc.).

PCR. A total of $1 \mu 1$ template cDNA, $45 \mu 1$ PCR SuperMix (Invitrogen; Thermo Fisher Scientific, Inc.) and 10 pmol (total volume, $50 \mu \mathrm{l}$ ) of each of the following primers: mstn forward, 5'-AGTGGATCTAAATGAGGGCAGT-3' and reverse, 5'-GTT TCCAGGCGCAGCTTAC-3'; GAPDH forward, 5'-TCACCA TGGAGAAGGC-3' and reverse, 5'-GCTAAGCAGTTGGTG CA-3' were processed using a TP600 thermocycler (Takara Biotechnology Co., Ltd.). PCR products were confirmed by ethidium bromide staining following electrophoresis on $1 \%$ agarose gels. Quantitative analyses of band densities were performed using a Gel Doc XR imaging system (Bio-Rad Laboratories, Inc.).

$R T$ - $q P C R$. A total of $1 \mu 1$ template cDNA, $5 \mu 1$ SYBR-Green PCR master mix (Applied Biosystems; Thermo Fisher Scientific, Inc.) and $10 \mathrm{pmol}$ (total volume, $10 \mu \mathrm{l}$ ) of each primer aforementioned was amplified were performed using an ABI 7900 PCR system (Applied Biosystems; Thermo Fisher Scientific, Inc.). The following thermocycling conditions were used for qPCR: $3 \mathrm{~min}$ at $95^{\circ} \mathrm{C}$, followed by two-step PCR at $95^{\circ} \mathrm{C}$ for $15 \mathrm{sec}$ and $60^{\circ} \mathrm{C}$ for $60 \mathrm{sec}$ for 40 cycles, with fluorescence monitoring at the end of each elongation step. Relative mRNA expression of target genes was calculated using the 
$2^{-\Delta \Delta \mathrm{Cq}}$ method (26). GAPDH was utilized as the endogenous control.

In vitro transduction. Viral particles of shLenti-Mstn GFP were purchased from Sirion Biotech $\mathrm{GmbH}$ based on the shRNA-Mstn A. To identify shLenti-Mstn GFP expression, $0.5 \mu \mathrm{l}$ viral particles (VPs; $8 \times 10^{8} \mathrm{VP} / \mathrm{ml}$ ) were transduced into 293 T cells. At 3 days post-transduction, GFP expression in transfected cells was confirmed using a fluorescence microscope (Ti2U FL; Nikon Corporation) and photographed using NIS-Element software (version 5.01.00; Nikon Corporation).

Western blotting. Proteins were collected from differentiated C2C12 cells using RIPA lysis buffer (Biosesang) supplemented with a $1 \mathrm{X}$ protease/phosphatase inhibitor cocktail (cat. no. 5871; Cell Signaling Technology, Inc.). Cell lysates were centrifuged at $13,000 \times \mathrm{g}$ for $20 \mathrm{~min}$ at $4^{\circ} \mathrm{C}$ and the supernatants were used for quantification using the BCA method. A total of $15 \mu \mathrm{g} /$ lane of each sample was separated on a $12 \%$ SDS-PAGE gel and transferred to PVDF membranes. The membranes were blocked with $5 \%$ bovine serum albumin (cat. no. 0219989680; MP Biomedicals, LLC) in TBST [20 mM Tris- $\mathrm{HCl}$ (pH 8.0), $150 \mathrm{mM} \mathrm{NaCl}, 0.1 \%$ (v/v) Tween-20; all Sigma-Aldrich; Merck KGaA] for $1 \mathrm{~h}$ at room temperature. Then, membranes were incubated overnight at $4{ }^{\circ} \mathrm{C}$ with the following primary antibodies: Anti-GDF8 (cat. no. ab71808; Abcam; 1:1,000) and anti- $\beta$-actin (cat. no. ab8227; Abcam; 1:1,000). Subsequently, bound antibodies were incubated with horseradish peroxidase-conjugated anti-rabbit immunoglobulin G secondary antibodies (cat. no. 7074; Cell Signaling Technology, Inc.; 1:2,000) for $1 \mathrm{~h}$ at room temperature. Western blotting luminol reagent (Santa Cruz Biotechnology, Inc.) was used to detect proteins. Integrated optical densities of the immunoreactive protein bands were measured using ImageJ software (version 1.8.0_112; National Institutes of Health).

Prepared mouse model. All animals were tested in a blinded manner. After administration, rotarod test, grip strength, MRI, NCS data acquisition, gait analysis and muscle isolation were performed separately by different researchers without information regarding the treatments. Animals were managed with strict adherence to protocols approved by the Institutional Animal Care and Use Committee of the Samsung Medical Center (approval no. SMC-20160507001). The mice were maintained in an environment of an illuminance of 300 lux, 30-70\% humidity and at $21^{\circ} \mathrm{C}\left( \pm 2^{\circ} \mathrm{C}\right)$. The mice were fed a normal diet, and access to food and water was free. The dark/light cycle was performed through dimming control at 08:00 am and 06:00 pm. C22 mice [B6;CBACa-Tg(PMP22) $\mathrm{C} 22 \mathrm{Clh} / \mathrm{H}]$ were purchased from MRC Harwell Institute. A total of 24 C22 and its wild-type littermate (WT) mice were used. According to a previous study, myelination in rodents begins on postnatal day 6 , and thus treatment for demyelinating neuropathy was conducted on postnatal day 6 (7). Therefore, administration of shLenti-Mstn or shLenti-mock was initiated on postnatal day 90 (P90) in C22 mice, which represented the phenotypic expression of CMT.

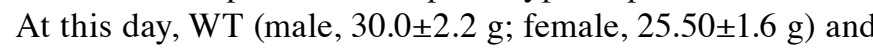
C22 (male, 29.6 $\pm 1.1 \mathrm{~g}$; female, $24.0 \pm 1.2 \mathrm{~g}$ ) were weighed. Thereafter, rotarod test, grip strength, electrophysiological study, magnetic resonance imaging and gait analysis were performed in all groups of mice at postnatal day 120 (P120). Subsequently, mice were sacrificed for muscle isolation. The mice were divided into the following groups: Mock (male, $n=4$; female, $n=3$ ) and shLenti-Mstn A (male, $n=7$; female, $n=3)$.

Mouse genotyping. Tail biopsies $(0.5 \mathrm{~cm})$ of mice at P24 were incubated at $55^{\circ} \mathrm{C}$ for $5-6 \mathrm{~h}$ in $150 \mu \mathrm{l}$ Direct PCR Reagent (cat. no. 102; FIAT International Ltd.) containing $200 \mu \mathrm{g} / \mathrm{ml}$ proteinase K (cat. no. p6556; Sigma-Aldrich; Merck KGaA). Following centrifugation at $300 \mathrm{x} g$ for $1 \mathrm{~min}$ at room temperature, the supernatant was inactivated at $85^{\circ} \mathrm{C}$ for $45 \mathrm{~min}$. Subsequently, the supernatant was diluted with deionized water at $1 / 10$, and $1 \mu 1$ supernatant was used as a template gDNA for PCR. Genotyping of C22 mice was performed using a pair of primers that amplify mouse and human cDNA. The typical PCR sample consisted of a $25 \mu \mathrm{l}$ volume containing 5 pmol primers for Yeast artificial chromosome (YAC; forward,5'-GCTACTTGGAGCCACTAT CGAGACGCGAT-3' and reverse, TGATAAATTAAAGTC TTGCGCCTTAAACC-3') and a control pair of primers for amplifying CD79b as a wild-type allele [IC1250; forward, 5'-GAGACTCTGGCTACTGATCC-3' and reverse, 5'-CCT TCAGCAAGAGCTGGGGAC-3' (27)]. The reaction also contained Hifi mixture (cat. no. 12532016; Thermo Fisher Scientific, Inc.) according to the manufacturer's protocol. The following PCR conditions were applied: Initial denaturation for $5 \mathrm{~min}$ at $94^{\circ} \mathrm{C}$; cyclic denaturation for $30 \mathrm{sec}$ at $94^{\circ} \mathrm{C}$, annealing for $30 \mathrm{sec}$ at $58^{\circ} \mathrm{C}$ and elongation for $1 \mathrm{~min}$ at $72^{\circ} \mathrm{C}$ for 35 cycles; and elongation for $5 \mathrm{~min}$ at $72^{\circ} \mathrm{C}$. PCR amplification products were analyzed by agarose gel electrophoresis as aforementioned.

In vivo transduction. Mice were injected with $120 \mu \mathrm{l}$ $\left(8 \times 10^{8} \mathrm{VP} / \mathrm{ml}\right)$ shLenti-Mstn or $120 \mu \mathrm{l} \mathrm{PBS}$ at the gastrocnemius muscle (GA) and rectus femoris (RF) sites into hind muscles using $1 \mathrm{ml}$ insulin syringe (cat. no. 328820; Becton, Dickinson and Company). As $120 \mu \mathrm{l}$ is a large quantity compared with muscle volume, administration was divided into three injections of $40 \mu \mathrm{l}$ with $10 \mathrm{~min}$ intervals. Doses were calculated using an in vitro assay $(20-100 \mathrm{pmol} / \mathrm{ml}$; cat. no. 631235; Clonetech Laboratories, Inc.) according to the manufacturer's protocol.

Muscle isolation. To evaluate muscle weight and evaluate mRNA expression levels at injection sites (GA and RF) of C22 transgenic mice administered with lentivirus, mice were euthanized at P120 at the end of the experiment. All mice euthanasia was performed by appropriately trained personnel approved by the Institutional Animal Care and Use Committee of the Samsung Medical Center (approval no. SMC-20160507001). Mice were euthanized by slow (30\% per minute) displacement of chamber air with compressed $\mathrm{CO}_{2}$ gas for $5 \mathrm{~min}$ in a $\mathrm{CO}_{2}$ chamber, and decapitation was performed to confirm complete irreversible euthanasia. Subsequently, each tissue of all groups was separated in a blinded manner by an experienced researcher. The isolated muscle was weighed using a microbalance and mRNA was isolated from the tissues as aforementioned. 
Rotarod performance test. To evaluate the motor coordination and balance of all groups of mice, mice performed the rotarod performance test by gradual acceleration on a $3-\mathrm{cm}$ horizontal rotating rod at a maximum speed of $2 \mathrm{~m} / \mathrm{min}$. Prior to the experiment, mice were pretrained for 3 days for acclimation. Performance was measured for a maximum of $5 \mathrm{~min}$.

Grip strength test. Grip strength of hind limbs in all groups of mice was measured every other day using a Grip Strength Meter (Ugo Basile S.R.L). Maximal forces of three trials were averaged and averaged values were divided by body weight.

Electrophysiological study. To assess electrophysiological status in mice groups of mock and shMstn-A, a nerve conduction study (NCS) was performed as previously described (13). Briefly, mice were initially anesthetized with $\mathrm{CO}_{2}$ gas and maintained with $1.5 \%$ isoflurane supplied using a nose cone for the duration of the procedure. This is a common and mandatory procedure to examine electrophysiological functions in NCS $(12,13)$. The present study administered 10-30\% chamber $\mathrm{vol} / \mathrm{min}$ of $\mathrm{CO}_{2}$ gas used for the initiation of anesthesia and then changed to isoflurane $(2-3 \% ; \geq 1 \mathrm{l} / \mathrm{min})$ to reduce any potential health risk and obtain accurate results. Previous studies have reported that an increased catecholamine concentration due to $\mathrm{CO}_{2}$ exposure can cause a higher stress response (increased heart rate and blood pressure), compared with isoflurane exposure (28-31). This method is frequently required by the Institutional Animal Care and Use Committee of the Samsung Medical Center (approval no. SMC-20160507001) for any repeated measurements of electrophysiological experiments to reduce potential stress.

Fur from the distal back to the hind limbs was completely removed. NCS was performed using a Nicolet Viking Quest device (Natus Medical, Inc.). For sciatic nerve motor NCS, an active recording needle electrode was placed into the gastrocnemius muscle with the reference electrode on the tendon. The stimulating cathode was placed proximal to the recording electrode in the midline of the posterior thigh to obtain the distal response and $6 \mathrm{~mm}$ proximally in the medial gluteal region to obtain the proximal response. Compound motor action potentials amplitudes (CMAP) and motor nerve conduction velocities (MNCV) were determined.

Lower limb magnetic resonance imaging (MRI) studies. To evaluate the muscle size of all groups of mice including WT, mock and shMstn A, MRI was performed using a $1.5 \mathrm{~T}$ system (Siemens AG). Axial, mid-sagittal and coronal scout rapid acquisition with fast low angle shot imaging was used to localize the legs. Area analysis using MRI images was measured using Paravision software (version 6.0; Bruker Corporation).

Gait analysis. Mouse gait was analyzed in all groups of mice using a TSE system MotoRater 303030 instrument (TSE Systems). Prior to measurement, mice were trained to spontaneously walk through the passage of the experimental apparatus. Mice were allowed to make a free round trip, for about $10 \mathrm{~min}$, by going back and forth from the beginning to the end of the equipment passage (width, $4 \mathrm{~cm}$; length, $80 \mathrm{~cm}$ ), at the same location for 4 days. At the end of each mouse's training, the inside of the passage was disinfected using $70 \%$ ethanol and dried. Video recordings were made to compare the changes in mouse pace throughout the daily training process. Prior to and to facilitate joint observation, the hair on the right hind flank of the mouse was flattened and white points were used to display each point of the iliac crest-hip-knee-ankle-foot. Video recordings began following confirmation that walking was performed normally compared with the 4-day training records. After the mouse walk measurements were completed, the interior of the aisle was disinfected using $70 \%$ ethanol and dried. The collected video recordings were analyzed to measure the time-dependent distance and joint angle changes using MotoRater and TSE System software (version 8.5.12; TSE Systems). Three indicators were selected as evaluation items: i) Stride length, which was the distance between the right front foot and the right rear foot with all feet touching the ground; ii) duty factor, which was the $\%$ of time the feet stayed in the air and on the ground; and iii) base of support, which was the gap with both forefeet touching the ground.

Statistical analysis. All data are presented as mean \pm SEM from at least three independent experiments. The statistical significance of the data presented in all figures was evaluated using one-way ANOVA with Tukey's post hoc multiple comparisons test. $\mathrm{P}<0.05$ was considered to indicate a statistically significant difference. Additionally, all animals were randomly assigned to each treatment and tested in a blinded manner.

\section{Results}

Determination of an optimal shRNA-Mstn system to suppress endogenous Mstn expression. Mstn expression regulates myoblast differentiation and proliferation by serving as a crucial negative activator during embryonic myogenesis (15-17).

The downregulation of Mstn expression using a shRNA system in the myogenic mouse $\mathrm{C} 2 \mathrm{C} 12$ line was investigated. Four different types of shRNA-Mstn (A, B, C and D) and mock (control shRNA) were transfected into $\mathrm{C} 2 \mathrm{C} 12$ cells. When examined by PCR, shRNA-Mstn A exhibited the highest significant Mstn suppressive effect $(>60 \% ; \mathrm{P}<0.01)$ compared with the other shRNA-Mstns and shRNA-Mstn A exhibited a higher significant suppressive effect compared with the mock group (Fig. 1A). Furthermore, shRNA-Mstn A decreased expression the endogenous protein level of Mstn ( $\geq 50 \%$; $\mathrm{P}<0.001$ ) compared with controls.

Following this, a Lenti-associated viral vector was constructed, which included shRNA-Mstn A and GFP (shLenti-Mstn A) for the in vivo study (Fig. 1B). Fluorescence imaging demonstrated GFP-expressing cells in 293T cells following shLenti-Mstn transduction (Fig. 1C). GFP expression was observed in most cells $(\sim 80 \%)$ and levels were similar to those in the mock group (Lenti-positive GFP). These results indicated that shLenti-Mstn-GFP may decrease Mstn expression in an in vivo model.

Improvement of muscle regeneration using shLenti-Mstn in C22 mice. The regulation of Mstn expression serves an important role in recovering skeletal muscle volume and mass (16-19). Whether treatment with shLenti-Mstn increased 

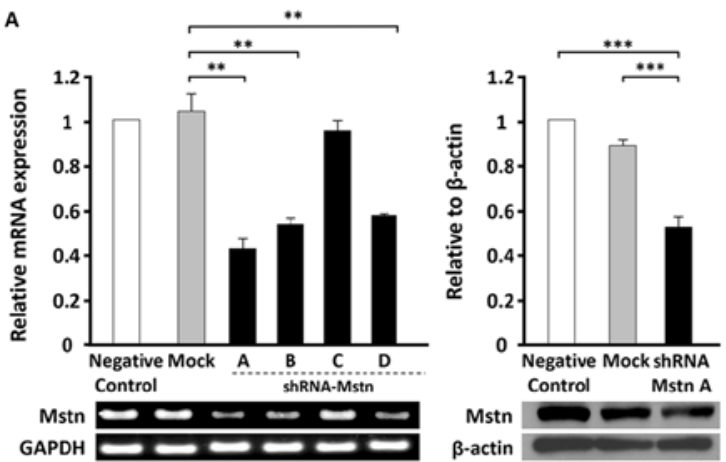

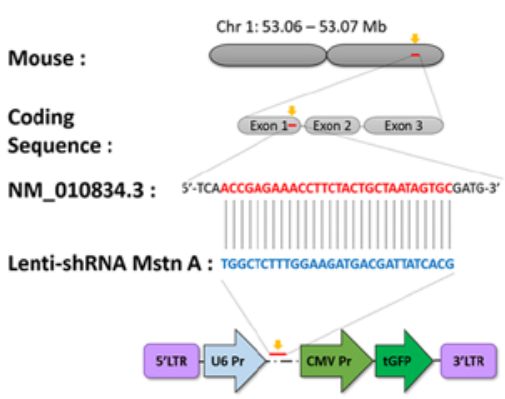

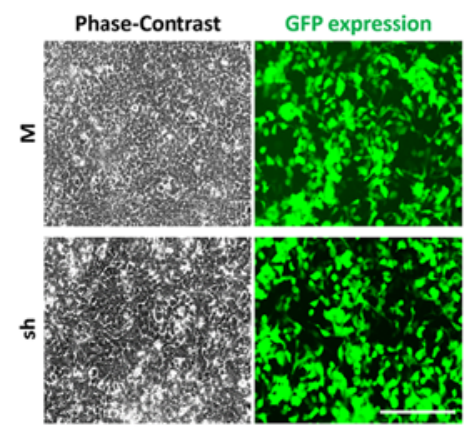

Figure 1. The optimal design of shRNA mstn and construction of the shLenti-system. (A) PCR analysis of the inhibition levels of $m s t n$ gene expression by four different shRNAs (shRNA-Mstn A, B, C and D) compared with the negative control and mock groups (left). Western blotting following transfection of shRNA-Mstn A to analyze the expression of endogenous Mstn (right). (B) Construction of the Lenti-shRNA system with GFP expression. Co-expression of shRNA-Mstn A and GFP following transduction genomic integration. (C) Expression of shLenti-Mstn A-GFP in 293T cells post-transfection. Magnification, $\mathrm{x} 200$. Scale bar, $40 \mu \mathrm{m} .{ }^{* *} \mathrm{P}<0.01$. ${ }^{* * *} \mathrm{P}<0.001$. Mstn, myostatin; GFP, green fluorescent protein; Chr, chromosome; Pr, promoter; CMV, cytomegalovirus; LTR, long terminal repeat; W, wild-type; M, mock; sh, shLenti-Mstn A.

A

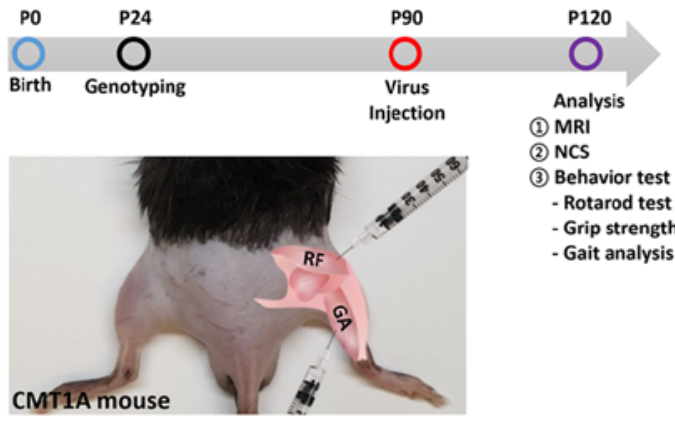

C
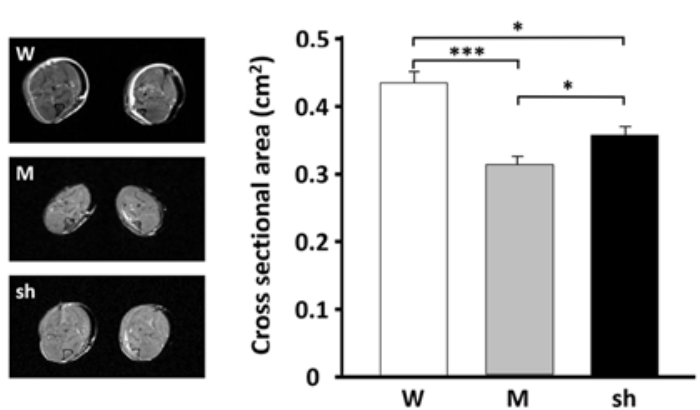
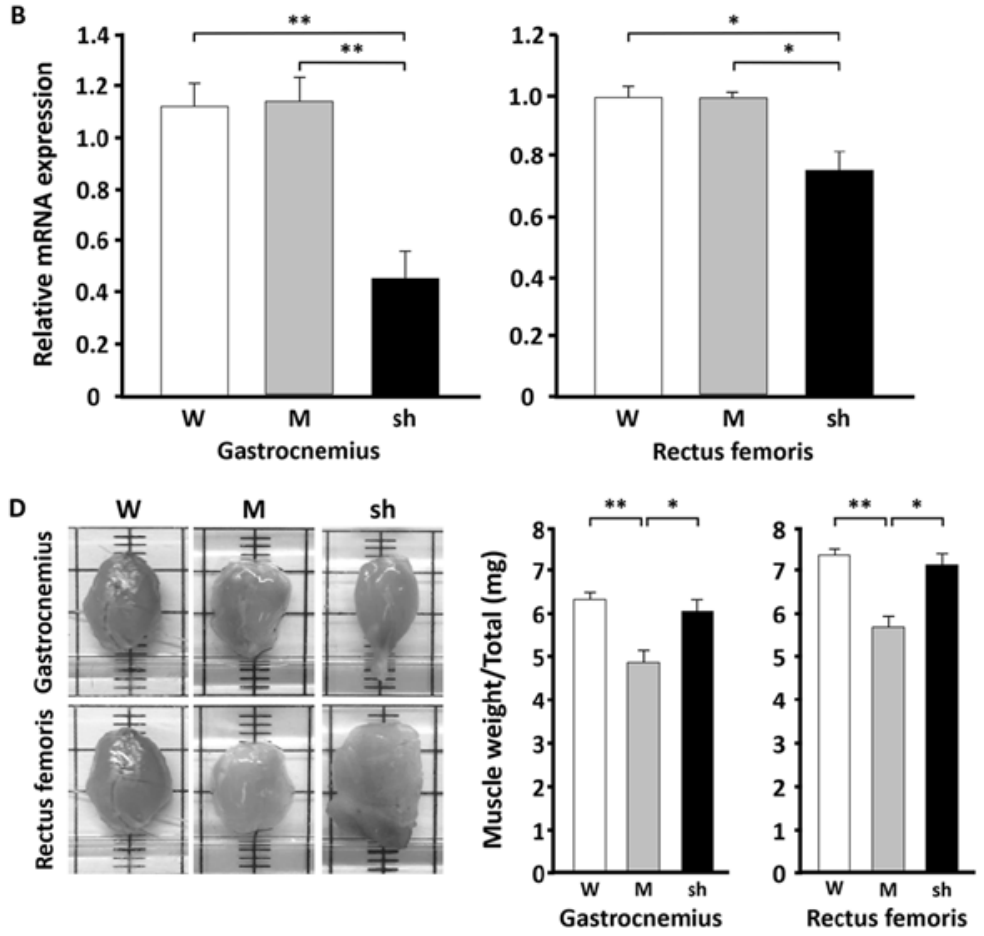

Figure 2. In vivo efficacy of shLenti-Mstn A treatment in CMT1A mice. (A) Schematic diagram of the in vivo study using the CMT1A mouse (C22) model and sites of infection by shLenti-Mstn in the hind limb of mice. (B) Reverse transcription-quantitative PCR of $m s t n$ expression levels at day 30 post-infection at two different sites, GA and RF, in the hind limb. (C) MRI images of the hind limb muscle area (left) in three different mice and quantification (right)(n=3). (D) Images of the GA and RF muscle mass (left) and quantification (right). WT (male $n=3$, female $n=4$ ), mock (male $n=4$, female $n=3$ ) and shLenti-Mstn A (male $\mathrm{n}=6$, female $\mathrm{n}=3$ ) groups were analyzed. ${ }^{*} \mathrm{P}<0.05,{ }^{* *} \mathrm{P}<0.01$ and ${ }^{* * *} \mathrm{P}<0.001$. Mstn, myostatin; GA, gastrocnemius; RF, rectus femoris; MRI, magnetic resonance imaging; TG, transgenic; W, wild-type; M, mock; sh, shLenti-Mstn A; P, postnatal; NCS, nerve conduction study; CMT1A, Charcot-Marie-Tooth disease type $1 \mathrm{~A}$.

muscle volume and mass in C22 mice with hind-limb muscle atrophy was investigated. The current study attempted to increase skeletal muscle volume and mass by injecting shLenti-Mstn A into two different sites, GA and RF of the hind limb, in C22 mice on postnatal day 90 (Fig. 2A).

Previous studies have demonstrated that $\mathrm{C} 22$ mice exhibit demyelinating neuropathy on P6 and muscle atrophy on P30 $(3,4,8)$. mRNA of the muscles from GA and RF of the hind limbs were isolated on P120. The results of RT-qPCR demonstrated that Mstn expression in the hind limb was significantly reduced (by $46.9 \%, \mathrm{P}<0.01$ ) by shLenti-Mstn compared with the wild-type (WT) and mock groups (Fig. 2B). Furthermore, shLenti-Mstn decreased the level of endogenous Mstn in the C22 mice following long-term transgene expression (Fig. 2B). MRI revealed that shLenti-mock (muscle atrophy, $0.312 \pm 0.012 \mathrm{~cm}^{2}$ ) caused a $\sim 27 \%$ decrease $(\mathrm{P}<0.001)$ in the cross sectional areas of hind limb volume in C22 mice compared with WT mice (muscle atrophy, $0.429 \pm 0.018 \mathrm{~cm}^{2} ;$ Fig. 2C). In contrast, shLenti-Mstn A (muscle atrophy, 0.355 $\pm 0.014 \mathrm{~cm}^{2}$ ) exhibited a significantly increased cross sectional area of the hind limb 

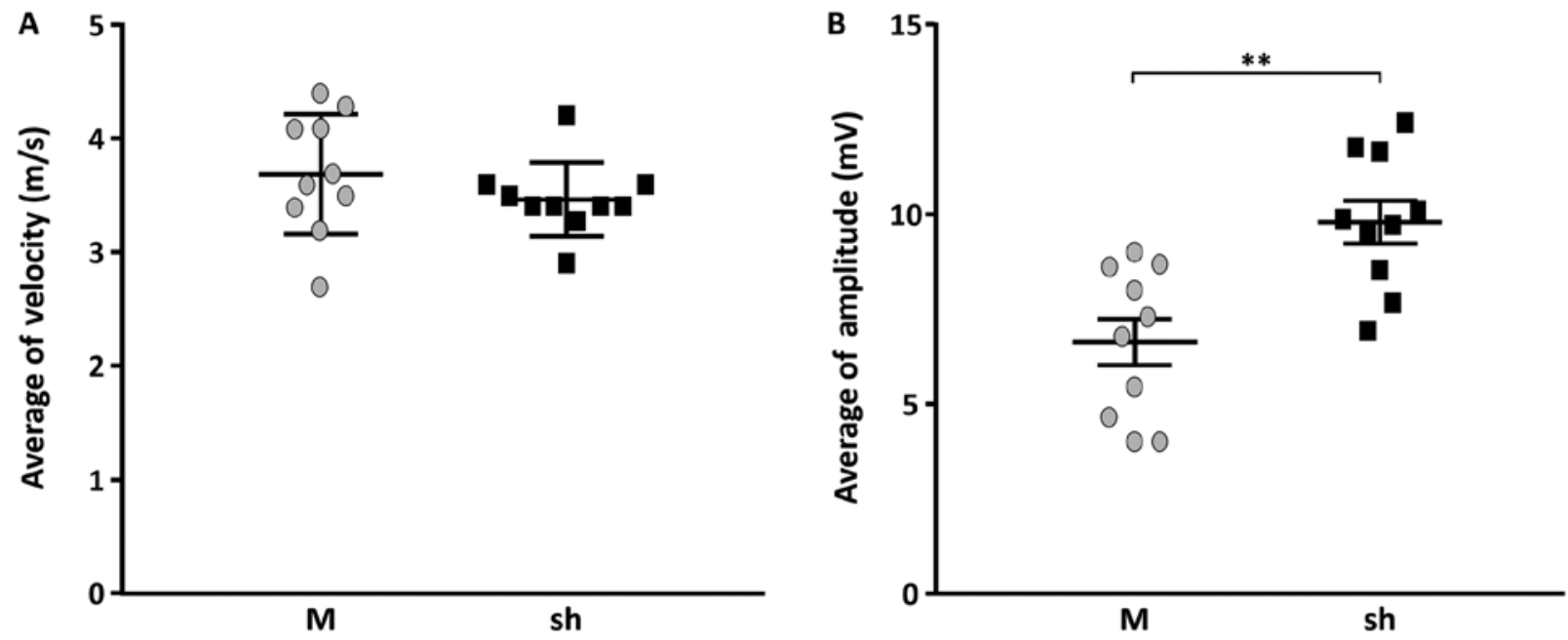

Figure 3. Electrophysiological parameters of motor nerves in hind limbs following shLenti-Mstn A treatment in C22 mice. (A) Average of velocity for NCV and (B) average of amplitude for CMAP were compared between the mock $(\mathrm{n}=7)$ and shLenti-Mstn A groups ( $\mathrm{n}=7 /$ group). ${ }^{* *} \mathrm{P}<0.01$. Mstn, myostatin; M, mock; sh, shLenti-Mstn A; NCV, nerve conduction velocity; CMAP, compound muscle action potential.

compared with the shLenti-mock group $(\mathrm{P}<0.05)$. Additionally, whether muscle mass was altered in shLenti-Mstn A-treated C22 mice was investigated by measuring the masses of GA and RF in each group. Following excision, muscle weight was significantly higher in the shLenti-Mstn A group (GA, $6.09 \pm 0.25 \mathrm{mg}$; RF, $7.11 \pm 0.48 \mathrm{mg}$ ) mice compared with mock (GA, $4.89 \pm 0.27 \mathrm{mg}$; RF, $5.51 \pm 0.47 \mathrm{mg}$ ) and WT (GA, $6.38 \pm 0.14 \mathrm{mg}$; RF, $7.35 \pm 0.29 \mathrm{mg}$ ) groups (Fig. 2D). These results demonstrated that shLenti-Mstn increased the muscle mass of both the GA and RF of the hind limb in C22 mice.

Enhancement of the electrophysiology in hind limbs following muscle regeneration. Nerve electrophysiology changes in the hind limb following shLenti-Mstn treatment were examined. C22 mice exhibiting demyelinating neuropathies, including abnormal MNCV and CMAP caused by human PMP22 gene overexpression (14,32-34). Following shLenti-Mstn A injections into the hind limbs of C22 mice, electrophysiological patterns were improved in the shLenti-Mstn A group compared with the mock group (Fig. 3). However, the NCS demonstrated that MNCV in the shLenti-Mstn A group (3.4 $\pm 0.4 \mathrm{~m} / \mathrm{sec})$ was not significantly different compared with the mock group $(3.7 \pm 0.5 \mathrm{~m} / \mathrm{sec}$ ) (Fig. 3A). In contrast, CMAP was significantly increased by $\sim 23 \%$ in the shLenti-Mstn A group $(9.9 \pm 0.7 \mathrm{mv})$ compared with the mock group $(6.7 \pm 0.6 \mathrm{mv})(\mathrm{P}<0.01$; Fig. 3B). Thus, these results indicated that the increased muscle mass improved CMAP in the hind limb of C22 mice injected with shLenti-Mstn A.

Regenerated hind-limb muscle improves locomotor coordination in C22 mice. Whether shLenti-Mstn A injections led to the improvement of locomotor coordination of $\mathrm{C} 22$ mice due to an increase in muscle mass was investigated. Behavioral test results, including those of the rotarod, grip strength and gait analysis were analyzed. This evaluation was used to determine locomotor coordination during mouse movement $(13,14)$.

C22 mice are reported to have unsteady gaits and muscle weakness caused by demyelinating peripheral neuropathies (32). In the current study, behavioral evaluations were measured on day 30 following injections of shLenti-Mstn A and a mock viral particle. As determined by the rotarod test (shLenti-mock, $2.8 \pm 0.49 \mathrm{sec}$; shLenti-Mstn A, $14.17 \pm 2.71 \mathrm{sec}$; Fig. 4A) and grip strength test [shLenti-mock, $0.25 \pm 0.11$ gram force (gf); shLenti-Mstn A, 1.12 \pm 0.17 gf; Fig.4B], mobility in the hind limb $(>5$-fold, $\mathrm{P}<0.01)$ and grip strength $(>1.7$-fold, $\mathrm{P}<0.01)$ were significantly increased in shLenti-Mstn A-injected group compared with the mock group. However, increased behavioral values in the shLenti-Mstn A group were significantly lower compared with the WT group $(\mathrm{P}<0.001)$. Following this, gait improvement was examined using a MotoRater system, which analyzes walking in C22 mice. Numerous previous studies on ALS have used walking analysis with the DigiGait system in mice (21-23). In these studies, mice exhibiting muscle atrophy demonstrated shortened stride and increased swing of the hind legs.

In the current study, swinging and walking in three different mice was measured using the MotoRater system. The results demonstrated that mock mice exhibited significantly lower stride-to-stride variability in both legs compared with the WT (SL-RH, $\mathrm{P}<0.001$; SL-LH, $\mathrm{P}<0.01$ ) and shLenti-Mstn A groups (SL-RH, $\mathrm{P}<0.05$; SL-LH, $\mathrm{P}<0.05$ ) (indicated by red arrows; Fig. 4C and D). Furthermore, shLenti-Mstn A mice demonstrated improved swinging and walking compared with mock mice (Fig. 4D). These results indicated that shLenti-Mstn A injections resulted in improved swinging and walking in the hind limbs of $\mathrm{C} 22$ mice.

\section{Discussion}

The current study investigated shRNA-based gene therapy using shLenti-Mstn in a mouse model of CMT1A. The results demonstrated that gait improved following therapy. shLenti-Mstn therapy reported a $>60 \%$ reduction of $m s t n$ mRNA in the myogenic $\mathrm{C} 2 \mathrm{C} 12$ mouse cell line. Furthermore, a single dose $\left[240 \mu \mathrm{l}\left(8 \times 10^{8} \mathrm{VP} / \mathrm{ml}\right) /\right.$ site] of shLenti-Mstn by intramuscular injection to $\mathrm{C} 22$ mice was accompanied with a $>40 \%$ reduction in mstn mRNA expression, which was observed on day 30 . 


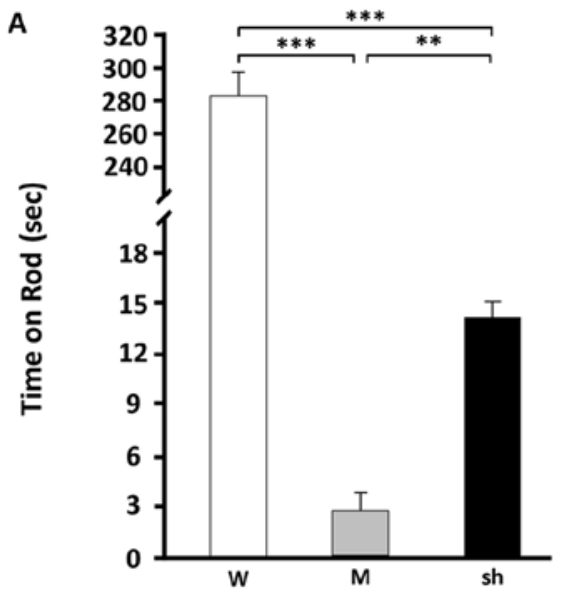

C
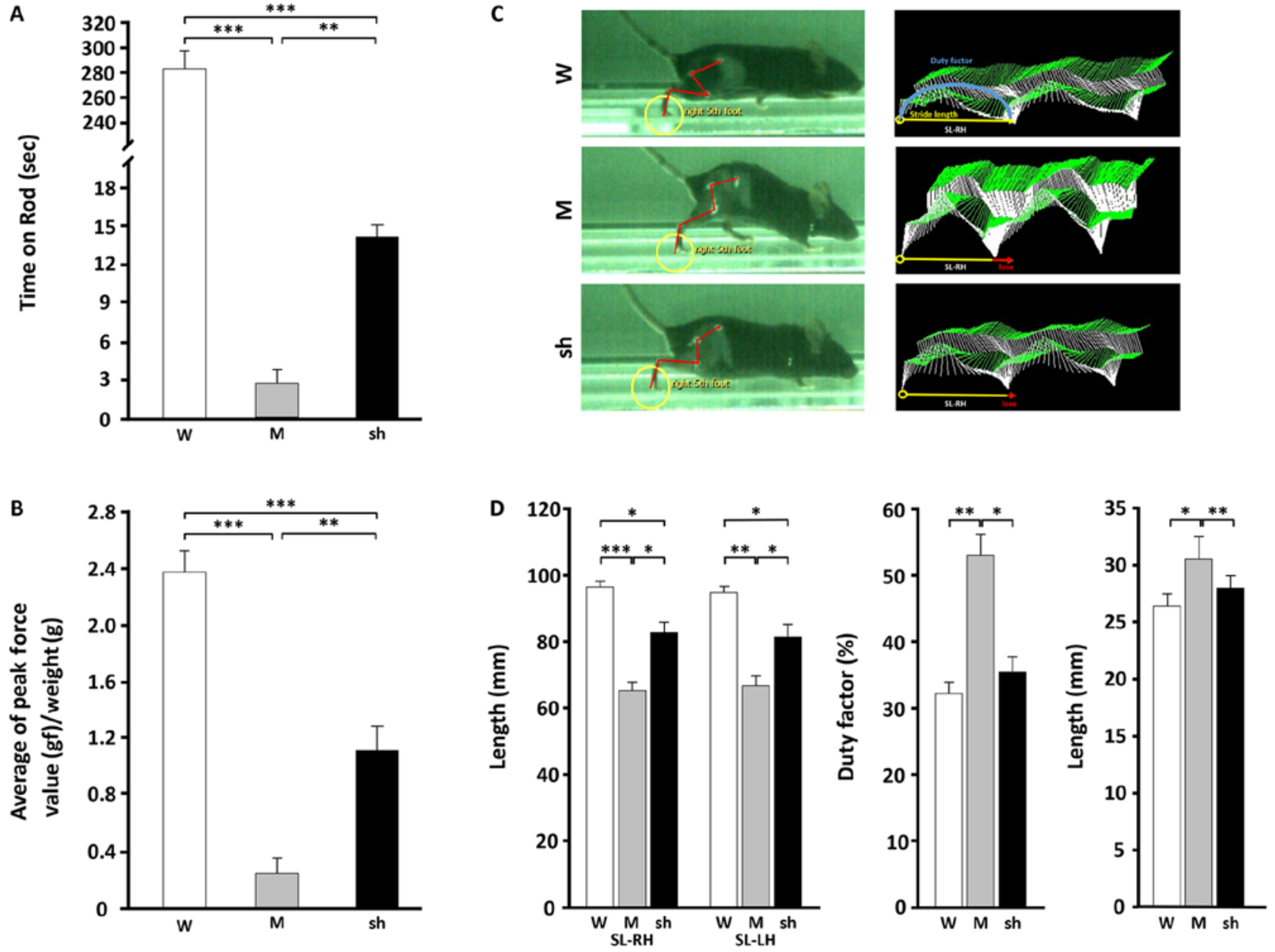

Figure 4. Effects of shLenti-Mstn A treatment on locomotion in C22 mice. (A) Rotarod test and (B) hind-limb grip strength following muscular injections of shLenti-Mstn A in the mock $(n=5)$ and shLenti-Mstn A ( $n=6)$ groups. (C) Real-time gait analysis of WT, mock and shLenti-Mstn A-treated mice ( $n=3 /$ group). Footprint colors were assigned by the software (foot, yellow circles; yellow line, distance between each footstep; red line, line connecting the pelvis, hip, knee, ankle and foot points together for analysis; red arrow, decreased step length compared with WT). (D) Quantitative analysis of SL (left panel), duty factor (middle panel) and base of support (right panel). $\mathrm{n}=3 /$ group. ${ }^{*} \mathrm{P}<0.05 .{ }^{* *} \mathrm{P}<0.01 .{ }^{* * * *} \mathrm{P}<0.001$. Mstn, myostatin; W, wild-type; M, mock; sh, shLenti-Mstn A; SL, stride length; $\mathrm{RH}$, right hind limb; LH, left hindlimb.

To support the results of $m s t n$ silencing, MRI imaging demonstrated that cross sectional area and muscle mass in GA significantly increased alongside the levels of CMAP. Consequently, the locomotion and gait pattern of $\mathrm{C} 22$ mice were improved. The shLenti-Mstn system used in the present study may be an important approach in the treatment of gait disorders and may increase the quality of life in patients with PNS conditions.

RNA-based gene-silencing methodologies represent an effective therapeutic strategy for the inhibition of specific target molecules in disease pathways. Due to the potential for increased specificity and reduced toxicity and side effects, RNA interference has the potential to surpass treatment with standard pharmacological drugs $(12,35)$.

Previous studies have demonstrated that non-coding RNAs, including microRNA and small interfering RNA, are potent therapeutic strategies for the treatment of neuropathies $(12,35)$. However, few studies have reported improvement in gait disorders due to muscle regeneration. Therefore, using the shMstn system, the current study attempted to increase the recovery of neurological disease-induced muscle atrophy. Among the four designed shMstn sequences targeting Mstn exon 1, shMstn A exhibited the highest suppression of Mstn in vitro with high transfection efficiency.

The regulation of Mstn expression is essential during myogenesis and skeletal muscle regeneration $(15,16)$. Following damage to the skeletal muscle, the regeneration process is activated and myoblasts sequentially differentiate into myocytes, myotubes and myofibers (15-20). In adulthood, MSTN is produced by skeletal muscles, circulated by the blood and acts to limit muscle fiber growth and it is a well-established endogenous inhibitor of myogenesis $(15,16,36)$. Furthermore, several studies have demonstrated that skeletal muscle satellite cells differentiate into myofibers during the regeneration mechanism in adult muscles $(15-20,36)$. This has led to speculation about the potential physiological roles of Mstn in peripheral neuropathies. Other previous studies have established animal models of muscle hypertrophy in various species through $m s t n$ gene knockouts or mutations (37-40). Notably, Lin et al (41) reported increased myogenesis and decreased adipogenesis through examination of intramuscular mechanisms in an $m s t n$-knockdown mouse model.

PNS diseases, including Guillain-Barrè syndrome and CMT, are accompanied by the demyelination of Schwann cells 
and axonal damage to sensory and motor nerve cells (1-5). In particular, motor nerve weakness gradually leads to muscle atrophy and gait disorders $(1,4,5)$. Following the onset of neuropathy, patients with CMT develop gait disorders due to the degeneration of motor nerves and skeletal muscle $(1,5)$. Treatments for skeletal muscle and the PNS are required to overcome these challenges. To the best of our knowledge, a direct correlation between CMT and Mstn muscle regeneration has not yet been reported. A recent study demonstrated that follistatin (a protein secreted by skeletal muscle)-based fusion protein binds and potently neutralizes Mstn (42). Furthermore, to the best of our knowledge, the current study is the first to use shLenti-Mstn therapy to treat gait disorder in a CMT disease mouse model.

Prepared shLenti-Mstn A was injected into two locations in the CMT1A disease mouse model. The two injection points, at the GA and RF, are the major muscles of the hind limb and are indispensable for walking (43). The results confirmed that shLenti-Mstn A significantly suppressed mstn gene expression and increased muscle volume and mass in the hind limbs in the CMT1A disease model compared with the mock group. Furthermore, the results of electrophysiological analysis revealed a statistically significant increase in CMAP compared with the mock group. However, there was no significant difference in MNCV between the shLenti-Mstn A group and the mock group. This indicated that in the neuropathy model, improved muscle mass ameliorated poor motor coordination, even if nerve function was not regenerated by shMstn therapy. The results suggested that shLenti-Mstn A treatment lead to enhanced muscle hypertrophy and functionality.

Furthermore, the present results demonstrated that increased muscle mass of the hind limb supported gait improvement in the $\mathrm{C} 22$ mouse model. The C22 mouse develops abnormal behavior at $\sim 2$ weeks of age and gradually exhibits abnormal gait (10-13). Previous studies focused on behavior assessments of locomotor coordination in CMT mouse models $(12,13,35)$.

MotoRater analysis provides more detailed information about mouse gait through real-time recording compared with footprint analysis $(21,22)$. Generally, footprint analysis can demonstrate the ability of mouse locomotion, including walking stability and body balance, which is based on the distance between footprints (44). Mancuso et al (23) focused on gait analysis using the DigiGait system for measuring the walking pattern in a mouse model of ALS.

The present study investigated the footprints and complete walking patterns in a mouse model of CMT1. Accordingly, shLenti-Mstn A treatment improved the unstable walking patterns in terms of stride length, duty factor and base of support. Additionally, during measurement of stride length in the right hind limb, stride was significantly decreased in the mock group compared with the WT group. The shLenti-Mstn A-injected mice demonstrated a significantly improved stride length and duty factor, and these values were increased in WT mice, although not significant for stride length. These results indicated that an increase in muscle mass may be able to mitigate walking patterns in the neuropathy model. Therefore, it was hypothesized that the regulation of Mstn activity improves gait problems caused by CMT disease.
In conclusion, it was hypothesized that shLentiMstn A-inducted Mstn suppression is associated with the growth and differentiation underlying CMT disease, which suppresses muscle contractions and induces muscle changes. As a result, Mstn suppression served an important role in CMT disease, thereby directly or indirectly affecting gait improvement. The regulation of muscle activity in neurological diseases alleviated the condition, which is associated with Mstn suppression. Further studies are required to identify the potential mechanism of action between the muscle and Mstn. Although lentivirus was used to knockdown Mstn expression in the present study, using lentivirus has limitations in clinical drug development as neuropathy-induced DNA integration, muscle loss and gait are regulated by regulating the expression of genes involved in muscle formation and development. Therefore, it is necessary to develop symptomatic therapeutic strategies for improving gait of CMT 1A by further investigating the mechanisms underlying neuromuscular development.

\section{Acknowledgements}

Not applicable.

\section{Funding}

The current study was supported by the National Research Foundation of Korea grants funded by the Korean government (grant nos. 2016 R1A5A 2007009, 2017 R1A2B 2004699, 2018 R1A4A 1024506 and 2018 R1C1B 6002586) and by the Korean Health Technology R\&D Project, Ministry of Health and Welfare (grant nos. HI14C 3484 and HI16C 0426).

\section{Availability of data and materials}

The datasets used and/or analyzed during the current study are available from the corresponding author on reasonable request.

\section{Authors' contributions}

YBH, JHK and BOC designed and managed the current study. HMD, GK and $\mathrm{HH}$ designed the current study and collected data. HMD, SHN, HWM, SBK, JH and KWC analyzed and interpreted data. HMD and JH drafted and critically revised the manuscript for important intellectual content. YBH, JHK and BOC supervised the study. All authors read and approved the final manuscript.

\section{Ethics approval and consent to participate}

The current study was approved by the Institutional Animal Care and Use Committees of Samsung Medical Center (Seoul, Korea; approval no. SMC-20160507001).

\section{Patient consent for publication}

Not applicable.

\section{Competing interests}

The authors declare that they have no competing interests. 


\section{References}

1. Skre H: Genetic and clinical aspects of Charcot-Marie-Tooth's disease. Clin Genet 6: 98-118, 1974.

2. Levitan IB and Kaczmarek LK: Intercellular Communication In: The Neuron: Cell and Molecular Biology 4th edition. Oxford University Press, New York, NY, pp153-328, 2015.

3. Rotthier A, Baets J, Timmerman V and Janssens K: Mechanisms of disease in hereditary sensory and autonomic neuropathies. Nat Rev Neurol 8: 73-85, 2012.

4. Rossor AM, Kalmar B, Greensmith L and Reilly MM: The distal hereditary motor neuropathies. J Neurol Neurosurg Psychiatr 83: 6-14, 2012

5. Harding AE and Thomas PK: The clinical features of hereditary motor and sensory neuropathy types I and II. Brain 103: 259-280, 1980.

6. Kim JY, Woo SY, Hong YB, Choi H, Kim J, Choi H, Mook-Jung I, Ha N, Kyung J, Koo SK, et al: HDAC6 inhibitors rescued the defective axonal mitochondrial movement in motor neurons derived from the induced pluripotent stem cells of peripheral neuropathy patients with HSPB1 mutation. Stem Cells Int 2016 9475981, 2016.

7. Fledrich R, Stassart RM and Sereda MW: Murine therapeutic models for Charcot-Marie-Tooth (CMT) disease. Br Med Bull 102: 89-113, 2012.

8. Robertson AM, Perea J, McGuigan A, King RH, Muddle JR, Gabreels-Festen AA, Thomas PK and Huxley C: Comparison of a new pmp22 transgenic mouse line with other mouse models and human patients with CMT1A. J Anat 200: 377-390, 2002.

9. Meyer zu Horste G, Prukop T, Liebetanz D, Mobius W, Nave KA and Sereda MW: Antiprogesterone therapy uncouples axonal loss from demyelination in a transgenic rat model of CMT1A neuropathy. Ann Neurol 61: 61-72, 2007.

10. Sereda MW, Meyer zu Horste G, Suter U, Uzma N and Nave KA: Therapeutic administration of progesterone antagonist in a model of Charcot-Marie-Tooth disease (CMT-1A). Nat Med 9: $1533-1537,2003$

11. Passage E, Norreel JC, Noack-Fraissignes P, Sanguedolce V, Pizant J, Thirion X, Robaglia-Schlupp A, Pellissier JF and Fontes M: Ascorbic acid treatment corrects the phenotype of a mouse model of Charcot-Marie-Tooth disease. Nat Med 10: 396-401, 2004

12. Lee JS, Kwak G, Kim HJ, Park HT, Choi BO and Hong YB MiR-381 attenuates peripheral neuropathic phenotype caused by overexpression of PMP22. Exp Neurobiol 28: 279-288, 2019.

13. Lee JS, Lee JY, Song DW, Bae HS, Doo HM, Yu HS, Lee KJ, Kim HK, Hwang H, Kwak G, et al: Targeted PMP22 TATA-box editing by CRISPR/Cas9 reduces demyelinating neuropathy of Charcot-Marie-Tooth disease type $1 \mathrm{~A}$ in mice. Nucleic Acids Res 48: 130-140, 2020.

14. Robaglia-Schlupp A, Pizant J, Norreel JC, Passage E, Saberan-Djoneidi D, Ansaldi JL, Vinay L, Figarella-Branger D, Levy N, Clarac F, et al: PMP22 overexpression causes dysmyelination in mice. Brain 125: 2213-2221, 2002.

15. McPherron AC, Lawler AM and Lee SJ: Regulation of skeletal muscle mass in mice by a new TGF-beta superfamily member. Nature 387: 83-90, 1997.

16. Lee SJ and McPherron AC: Regulation of myostatin activity and muscle growth. Proc Natl Acad Sci USA 98: 9306-9311, 2001.

17. Mariot V, Joubert R, Hourde C, Feasson L, Hanna M, Muntoni F, Maisonobe T, Servais L, Bogni C, Le Panse R, et al: Downregulation of myostatin pathway in neuromuscular diseases may explain challenges of anti-myostatin therapeutic approaches. Nat Commun 8: 1859, 2017.

18. Wagner KR, McPherron AC, Winik N and Lee SJ: Loss of myostatin attenuates severity of muscular dystrophy in $\mathrm{mdx}$ mice. Ann Neurol 52: 832-836, 2002.

19. Holzbaur EL, Howland DS, Weber N, Wallace KE, She Y, Kwak S, Tchistiakova LA, Murphy E, Hinson J, Karim R, et al: Myostatin inhibition slows muscle atrophy in rodent models of amyotrophic lateral sclerosis. Neurobiol Dis 23: 697-707, 2006

20. Hennebry A, Oldham J, Shavlakadze T, Grounds MD, Sheard P, Fiorotto ML, Falconer S, Smith HK, Berry C, Jeanplong F, et al: IGF1 stimulates greater muscle hypertrophy in the absence of myostatin in male mice. J Endocrinol 234: 187-200, 2017.

21. Dorman CW, Krug HE, Frizelle SP, Funkenbusch $S$ and Mahowald ML: A comparison of DigiGait ${ }^{\mathrm{TM}}$ and TreadScan ${ }^{\mathrm{TM}}$ imaging systems: Assessment of pain using gait analysis in murine monoarthritis. J Pain Res 7: 25-35, 2013
22. Xu Y, Tian N, Bai Q, Chen Q, Sun XH and Wang Y: Gait assessment of pain and analgesics: Comparison of the DigiGait ${ }^{\mathrm{TM}}$ and CatWalk $^{\mathrm{TM}}$ gait imaging systems. Neurosci Bull 35: 401-418, 2019.

23. Mancuso R, Olivan S, Osta R and Navarro X: Evolution of gait abnormalities in SOD1(G93A) transgenic mice. Brain Res 1406: 65-73, 2011

24. DiGiusto DL, Krishnan A, Li L, Li H, Li S, Rao A, Mi S, Yam P, Stinson S, Kalos M, et al: RNA-based gene therapy for HIV with lentiviral vector-modified CD34(+) cells in patients undergoing transplantation for AIDS-related lymphoma. Sci Transl Med 2: 36ra43, 2010

25. Maples PB, Kumar P, Yu Y, Wang Z, Jay C, Pappen BO, Rao DD, Kuhn J, Nemunaitis J and Senzer N: FANG Vaccine: Autologous tumor cell vaccine genitically modified to express GM-CSF and block production of furin. BioProcess J 8: 4-14, 2009.

26. Livak KJ and Schmittgen TD: Analysis of relative gene expression data using real-tie quantitative PCR and the 2(-Delta Delta C(T)) method. Methods 25: 402-408, 2001.

27. Hasenberg A, Hasenberg M, Männ L, Neumann F, Borkenstein L, Stecher M, Kraus A, Engel DR, Klingberg A, Seddigh P, et al: Catchup: A mouse model for imaging-based tracking and modulation of neutrophil granulocytes. Nat Methods 12: 445-452, 2015.

28. Boivin GP,Hickman DL, Creamer-Hente MA, Pritchett-Corning KR and Bratcher NA: Review of $\mathrm{CO}_{2}$ as a euthanasia agent for laboratory rats and mice. J Am Assoc Lab Anim Sci 56: 491-499, 2017.

29. Marquardt N, Feja M, Hünigen H, Plendl J, Menken L, Fink H and Bert B: Euthanasia of laboratory mice: Are isoflurane and sevoflurane real alternatives to carbon dioxide? PLoS One 13 e0203793, 2018

30. Makowska IJ and Weary DM: Rat aversion to induction with inhalant anaesthetics. Appl Anim Behav Sci 119: 229-235, 2009.

31. Leach MC, Bowell VA, Allan TF and Morton DB: Degrees of aversion shown by rats and mice to different concentrations of inhalational anaesthetics. Vet Rec 150: 808-815, 2002.

32. Verhamme C, King RH, ten Asbroek AL, Muddle JR, Nourallah M, Wolterman R, Baas F and van Schaik IN: Myelin and axon pathology in a long-term study of PMP22-overexpressing mice. J Neuropathol Exp Neurol 70: 386-398, 2011

33. Komyathy K, Neal S, Feely S, Miller LJ, Lewis RA, Trigge G, Siskind CE, Shy ME and Ramchandren S: Anterior tibialis CMAP amplitude correlations with impairment in CMT1A. Muscle Nerve 47: 493-496, 2013.

34. Stojkovic T: Hereditary neuropathies: An update. Rev Neurol (Paris) 172: 775-778, 2016.

35. Lee JS, Chang EH, Koo OJ, Jwa DH, Mo WM, Kwak G, Moon HW, Park HT, Hong YB and Choi BO: Pmp22 mutant allele-specific siRNA alleviates demyelinating neuropathic phenotype in vivo. Neurobiol Dis 100: 99-107, 2017.

36. Chal $\mathrm{J}$ and Pourquie O: Making muscle: Skeletal myogenesis in vivo and in vitro. Development 144: 2104-2122, 2017

37. McPherron AC and Lee SJ: Double muscling in cattle due to mutations in the myostatin gene. Proc Natl Acad Sci USA 94: 12457-12461, 1997.

38. Grobet L, Martin LJ, Poncelet D, Pirottin D, Brouwers B, Riquet J, Schoeberlein A, Dunner S, Menissier F, Massabanda J, et al: A deletion in the bovine myostatin gene causes the double-muscled phenotype in cattle. Nat Genet 17: 71-74, 1997.

39. Kambadur R, Sharma M, Smith TP and Bass JJ: Mutations in myostatin (GDF8) in double-muscled Belgian blue and piedmontese cattle. Genome Res 7: 910-916, 1997.

40. Grobet L, Poncelet D, Royo LJ, Brouwers B, Pirottin D, Michaux C, Menissier F, Zanotti M, Dunner S and Georges M: Molecular definition of an allelic series of mutations disrupting the myostatin function and causing double-muscling in cattle. Mamm Genome 9: 210-213, 1998.

41. Lin J, Arnold HB, Della-Fera MA, Azain MJ, Hartzell DL and Baile CA: Myostatin knockout in mice increases myogenesis and decreases adipogenesis. Biochem Biophys Res Commun 291: 701-706, 2002.

42. Pearsall RS, Davies MV, Cannell M, Li J, Widrick J, Mulivor AW, Wallner S, Troy ME, Spaits M, Liharska K, et al: Follistatin-based ligand trap ACE-083 induces localized hypertrophy of skeletal muscle with functional improvement in models of neuromuscular disease. Sci Rep 9: 11392, 2019.

43. Bonnefoy A and Armand S: Normal Gait. In: Orthopedic Management of Children With Cerebral Palsy: A Comprehensive Approach. Nova Science Publishers Inc., Hauppauge, NY, p567, 2015.

44. Chester VL, Biden EN and Tingley M: Gait analysis. Biomed Instrum Technol 39: 64-74, 2005 